\title{
Deterioration of exchange bias in CoO-Co bilayers by the roughness of the $\mathrm{ZnO}$ substrates
}

\author{
D. Stamopoulos ${ }^{\mathrm{a}}$, M. Zeibekis, E. Manios, N. Boukos and D. Niarchos
}

Institute of Advanced Materials, Physicochemical Processes, Nanotechnology and Microsystems, National Center for Scientific Research ‘Demokritos', 15310, Aghia Paraskevi, Greece

\begin{abstract}
The Exchange Bias (EB) effect is observed at the interface of Antiferromagnet/Ferromagnet $(\mathrm{AF} / \mathrm{FM})$ structures and depends on the interface roughness (IR). Until today, only low IR values, usually below $10 \mathrm{~nm}$, have been investigated. Here we investigate an extended range of IR through controlling the surface roughness (SR) of the employed substrates. We employ CoO/Co bilayers (thickness within 10-60 nm), a classic $\mathrm{AF} / \mathrm{FM}$ structure that exhibits intense $\mathrm{EB}$. $\mathrm{ZnO}$ was employed as the substrate in both film and bulk forms, enabling us to vary the SR up to $840 \mathrm{~nm}$. Our data reveal a strong relative decrease, ranging within 20$65 \%$, of both the shift $\mathrm{H}_{\text {shift }}{ }^{\mathrm{EB}}$ and coercive $\mathrm{H}_{\mathrm{c}}{ }^{\mathrm{EB}}$ fields upon increase of SR (IR), for both parallel and normal magnetic field-sample configurations. For the explanation of these findings we propose that in thin AF/FM structures deposited on rough substrates the local magnetization, $\mathbf{M}_{\mathbf{f}}$ of the FM is 'locked' mainly in-layer due to shape anisotropy, thus it is forced to follow the morphologically rough landscape of the substrate. This imposes misalignment between $\mathbf{M}_{\mathbf{f}}$, that is 'directionally random', and $\mathbf{H}_{\mathbf{e x}}$, that is 'directionally oriented'. This weakens the biasing potential of $\mathbf{H}_{\mathbf{e x}}$ on $\mathbf{M}_{\mathbf{f}}$ and reduces the relative macroscopic parameters $\mathrm{H}_{\text {shift }}{ }^{\mathrm{EB}}$ amd $\mathrm{H}_{\mathrm{c}}{ }^{\mathrm{EB}}$.
\end{abstract}

\section{Introduction}

Exchange bias (EB) coupling [1] is primarily an interface effect observed in Antiferromagnet/Ferromagnet $(\mathrm{AF} / \mathrm{FM})$ structures that is used in a wide spectrum of applications of both technological and basic research interest [1]. Referring to technological applications, it is commonly used in FM/NM/FM and FM/IN/FM trilayers (where NM and IN stand for normal metal and insulator, respectively) to 'pin' the magnetization of the one outer FM layer. By using the EB effect in such structures the relative magnetization configuration (homo-parallel or anti-parallel) of the outer FM layers can be controlled. In this way, the magnetoresistance of the complete $\mathrm{AF} / \mathrm{FM} / \mathrm{NM} / \mathrm{FM}$ and $\mathrm{AF} / \mathrm{FM} / \mathrm{IN} / \mathrm{FM}$ structures can be switched between two distinct states, thus enabling the utilization of these structures in devices such as magnetic field sensors and memory units [2-5]. Regarding basic research, the EB effect can be used in investigations on hybrid structures between FM and superconducting (SC) constituents that are generally considered as incompatible since the exchange field of the FM is detrimental to the singlet pairing of electrons that is at play in conventional low- $\mathrm{T}_{\mathrm{c}} \mathrm{SC}$. However, in recent years hybrid $\mathrm{AF} / \mathrm{FM} / \mathrm{SC} / \mathrm{FM}$ trilayers have attracted much interest due to their interesting transport properties [6-9]. In brief, the critical current, $j_{c}$ and the critical temperature, $T_{c}$ of the $\mathrm{SC}$ interlayer can be controlled by the relative magnetization configuration of the outer FM layers, whether we refer to in-plane (superconducting spin valve effect based on the exchange fields) or out-of-plane (superconducting magnetoresistance effect based on the stray fields), in respect to the hybrid surface [7-9]. Recent experiments proved that the EB mechanism can be used to control the transverse magnetostatic coupling of the outer FM layers through controlling the stray fields that pierce the SC interlayer, thus ultimately controlling the transport properties of those hybrid structures [7-9].

All applications of the EB effect discussed above depend on the interface roughness (IR) between the FM layers and the NM, IN or SC interlayer, since increased IR can motivate out-of-plane magnetization processes that ultimately can promote the magnetostatic coupling of the outer FM layers through the interlayer whether it is NM, IN or SC [9-11]. Thus, investigations of the influence of the IR on the EB effect are of interest to reveal its contribution to each of these phenomena.

Up to now, many studies have focused on low IR, usually below $10 \mathrm{~nm}$ ([1] and references therein). Here we investigate an extended range of IR through controlling the surface roughness (SR) of the employed substrates. The study is conducted on $\mathrm{CoO} / \mathrm{Co}$ bilayers, a well studied AF/FM model system that exhibits intense EB. Zinc oxide $(\mathrm{ZnO})$ was employed as the substrate in both film and bulk forms, enabling us to vary the SR up to $840 \mathrm{~nm}$.

\footnotetext{
${ }^{a}$ Corresponding author: densta@ims.demokritos.gr
} 


\section{Methods}

$\mathrm{ZnO}$ substrates of variable SR (mean surface roughness denoted as $\left\langle\mathrm{S}_{\mathrm{a}}>\right.$ ) were prepared in bulk (pellet) and layered (film) forms by means of sintering and e-beam evaporation on $\mathrm{SiO}_{2}$ substrates, respectively. The SR of the $\mathrm{ZnO}$ bulk substrates was systematically varied through sintering. Starting powder (99.7\% purity) was initially dry pressed in pellet form under 100 Tones for 2 minutes and was sintered at different temperatures for 12 hours in air. Obviously, by changing the SR of the substrate we vary the IR of the AF/FM bilayer, that is deposited on top.

Co films with thickness ranging within 10-60 nm were rf-sputtered at 30 Watt under $3 \times 10^{-3}$ Torr of $99.999 \%$ pure $\mathrm{Ar}$ atmosphere on the $\mathrm{ZnO}$ substrates. The desired $\mathrm{CoO}$ AF layer was formed at the bottom part of the Co film during the deposition process through the absorption of $\mathrm{O}_{2}$ originating from the $\mathrm{ZnO}$ substrates.

The SR was systematically estimated from Atomic Force Microscopy (AFM) data that were acquired by means of a scanning probe microscope Solver PRO [NTMDT Co, Moscow, Russia] having a 100x100x5 $\mu^{3}$ xyz-scanner hosted on an active vibration isolation table Halcyonics MOD-1M Plus [Halcyonics $\mathrm{GmbH}$, Goettingen, Germany]. Our measurements were performed in the non-contact scanning mode with NCHR cantilevers that end to silicon nitride tips [Nano and More $\mathrm{GmbH}$, Wetzlar, Germany] having the nominal parameters, spring constant $=42 \mathrm{Nm}^{-1}$ and resonance frequency $=320 \mathrm{kHz}$. The optimum imaging results were obtained for scanning parameters: line frequency $=1-2 \mathrm{~Hz}$, area $=0.5 \times 0.5-50 \times 50 \mu \mathrm{m}^{2}$ and lines per image $=256-512$.

Detailed magnetization data were obtained using a SQUID unit [Quantum Design, San Diego, CA, USA]. Both parallel and normal configurations of the external magnetic field, $\mathbf{H}_{\mathbf{x}}$ in respect to the sample surface were investigated. Referring to the EB mechanism both virgin and biased loops were obtained by cooling from room temperature under zero and a sufficiently high magnetic field, respectively.

\section{Results and discussion}

Figure 1 shows the dependence of the mean surface roughness, $<\mathrm{S}_{\mathrm{a}}>$ on the sintering temperature, $\mathrm{T}_{\text {sin }}$. We see that the SR, as calculated from detailed AFM measurements (see figures 5(a)-5(c), below), exhibits an abrupt increase from $<\mathrm{S}_{\mathrm{a}}>=120 \mathrm{~nm}$ at $\mathrm{T}_{\sin }=800{ }^{\circ} \mathrm{C}$ to $<\mathrm{S}_{\mathrm{a}}>=840 \mathrm{~nm}$ at $\mathrm{T}_{\sin }=1400{ }^{\circ} \mathrm{C}$. The respective value of a $200 \mathrm{~nm}$ thick $\mathrm{ZnO}$ films that were used as reference was $<\mathrm{S}_{\mathrm{a}}>=33 \mathrm{~nm}$ (black circle in figure 1).

Since it is well known that the EB effect is strongly reduced upon increase of the FM layer thickness as $\mathrm{H}_{\text {shift }}{ }^{\mathrm{EB}} \sim 1 / \mathrm{d}_{\mathrm{FM}}[1]$ in this study we examined relatively thin Co films, with thickness ranging within 10-60 nm, aiming to preserve a noticeable effect even for the case of increased IR investigated here. We examined both the normal and parallel magnetic field-sample configurations. Fig. 1. Dependence of the mean surface roughness, $<\mathrm{S}_{\mathrm{a}}>$ on the sintering temperature, $\mathrm{T}_{\mathrm{sin}}$ for as prepared $\mathrm{ZnO}$ bulk pellets. The black circle represents the mean surface roughness of a $\mathrm{ZnO}$ film of thickness $200 \mathrm{~nm}$. The solid line is guide to the eye.

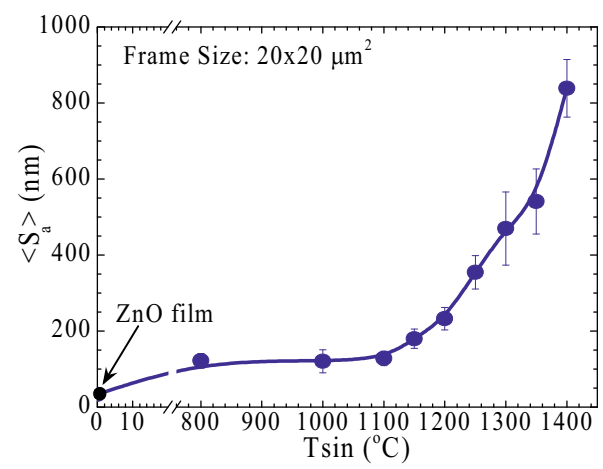

Figures 2(a) and 2(b) show representative isothermal magnetization loops at $\mathrm{T}=10 \mathrm{~K}$, for the parallel magnetic field-sample configuration, for Co thickness of $10 \mathrm{~nm}$ and $60 \mathrm{~nm}$, respectively. Both the virgin and biased states are presented for the $\mathrm{ZnO}$-film substrate, while only the biased state is shown for the two $\mathrm{ZnO}$-bulk substrates sintered at the lowest, $\mathrm{T}_{\sin }=800{ }^{\circ} \mathrm{C}$ and highest, $\mathrm{T}_{\text {sin }}=1400$ ${ }^{\circ} \mathrm{C}$ temperatures investigated here having the lowest, $<\mathrm{S}_{\mathrm{a}}>=120 \mathrm{~nm}$ and highest, $<\mathrm{S}_{\mathrm{a}}>=840 \mathrm{~nm}$ SR mean


values, respectively.

Fig. 2. Isothermal magnetization loops measured at $\mathrm{T}=10 \mathrm{~K}$ for the parallel magnetic field-sample configuration for $\mathrm{Co}$ thickness (a) $10 \mathrm{~nm}$ and (b) $60 \mathrm{~nm}$. In both cases the virgin and biased loops are presented for the $\mathrm{ZnO}$-film, while only the biased loops are shown for the $\mathrm{ZnO}$-bulk sintered at $\mathrm{T}_{\sin }=800{ }^{\circ} \mathrm{C}$ and $\mathrm{T}_{\mathrm{sin}}=1400^{\circ} \mathrm{C}$.

By comparing the virgin and biased loops, a regular negative EB shift, $\mathrm{H}_{\text {shift }}$ EB is observed, associated with the unidirectional magnetic anisotropy of the biased system in the opposite direction of the cooling field. Except for 
the behavior of $\mathrm{H}_{\text {shift }}{ }^{\mathrm{EB}}$ we have also analyzed the respective variation of the coercive field, $\mathrm{H}_{\mathrm{c}}^{\mathrm{EB}}$. From these data we see that both $\mathrm{H}_{\text {shift }}{ }^{\mathrm{EB}}$ and $\mathrm{H}_{\mathrm{c}}^{\mathrm{EB}}$ decrease with Co thickness, as expected [1] (note the different magnetic field range in figures 2(a) and 2(b)). In addition, both $\mathrm{H}_{\text {shift }}{ }^{\mathrm{EB}}$ and $\mathrm{H}_{\mathrm{c}}{ }^{\mathrm{EB}}$ progressively decrease as the $<\mathrm{S}_{\mathrm{a}}>$ of the substrate (and the respective IR of the $\mathrm{CoO} / \mathrm{Co}$ structure) increases. The magnetization data for the normal magnetic field-sample configuration are similar.

To quantify the influence of $<\mathrm{S}_{\mathrm{a}}>$ on these parameters we define the EB shift field as

$$
\mathrm{H}_{\text {shift }} \mathrm{EB}^{=-}\left(\mathrm{H}_{\mathrm{c}-}+\mathrm{H}_{\mathrm{c}+}\right) / 2
$$

and the EB coercive field as

$$
\mathrm{H}_{\mathrm{c}}^{\mathrm{EB}}=-\left(\mathrm{H}_{\mathrm{c}-}-\mathrm{H}_{\mathrm{c}+}\right) / 2
$$

where $\mathrm{H}_{\mathrm{c}-}$ and $\mathrm{H}_{\mathrm{c}+}$ are the coercive fields defined from the descending and ascending branches of the magnetization loop, respectively.
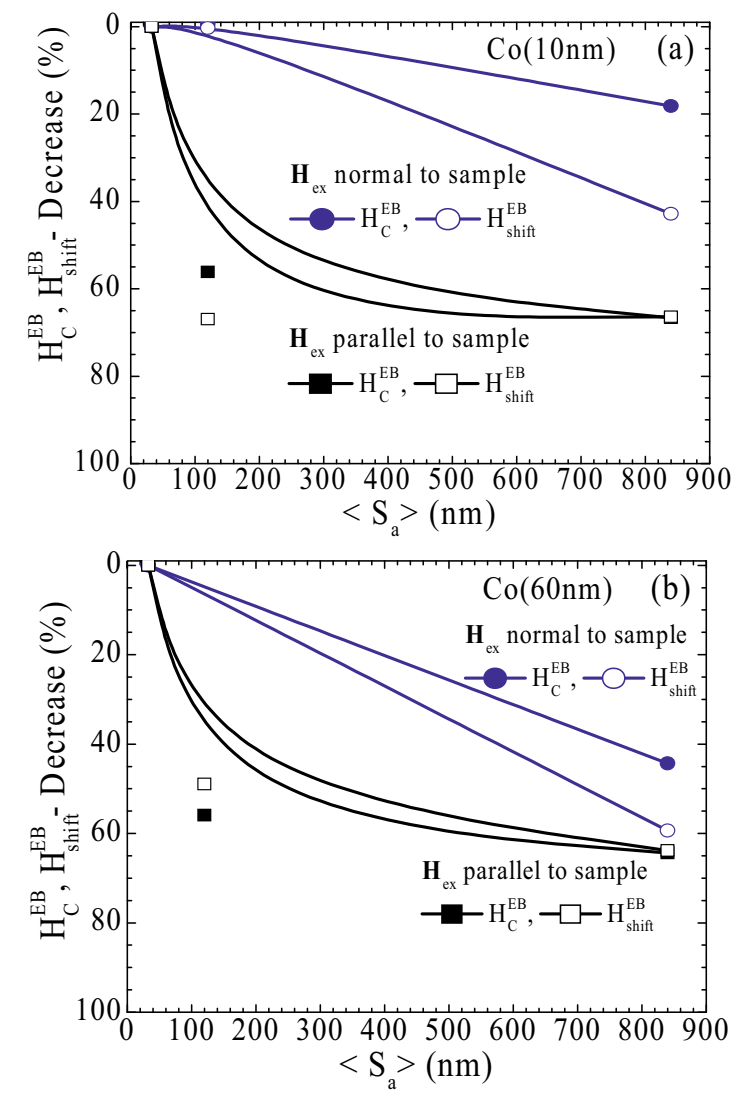

Fig. 3. Relative decrease of $\mathrm{H}_{c}{ }^{\mathrm{EB}}$ and $\mathrm{H}_{\text {shift }}{ }^{\mathrm{EB}}$ as a function of mean surface roughness, $<\mathrm{S}_{\mathrm{a}}>$ of the substrate for both parallel and normal magnetic field-sample configurations for $\mathrm{Co}$ thickness (a) $10 \mathrm{~nm}$ and (b) $60 \mathrm{~nm}$. The solid lines are guide to the eye.

The results for both the parallel and normal magnetic field-sample configurations are summarized in figures 3(a) and 3(b) for the Co films of $10 \mathrm{~nm}$ and $60 \mathrm{~nm}$, respectively. From these data we see a strong relative decrease of both $\mathrm{H}_{\text {shift }}{ }^{\mathrm{EB}}$ and $\mathrm{H}_{\mathrm{c}}{ }^{\mathrm{EB}}$ with the increase of IR induced by the increased $<\mathrm{S}_{\mathrm{a}}>$ of the $\mathrm{ZnO}$ substrates, for both parallel and normal $\mathbf{H}_{\mathbf{e x}}$. For the parallel magnetic field-sample configuration the relative decrease of both $\mathrm{H}_{\text {shift }}{ }^{\mathrm{EB}}$ and $\mathrm{H}_{\mathrm{c}}{ }^{\mathrm{EB}}$ does not seem to depend on the Co thickness exhibiting a very similar value of almost $65 \%$ for the maximum $<\mathrm{S}_{\mathrm{a}}>=840 \mathrm{~nm}$. On the contrary, for the normal magnetic field-sample configuration the relative decrease of $\mathrm{H}_{\text {shift }}$ EB is almost $45 \%$ for Co thickness of 10 $\mathrm{nm}$, while it is noticeably higher, $60 \%$ for Co thickness of $60 \mathrm{~nm}$. Referring to $\mathrm{H}_{\mathrm{c}}^{\mathrm{EB}}$ the relative decrease is almost $20 \%$ for Co thickness of $10 \mathrm{~nm}$, while it is again noticeably higher, $45 \%$ for Co thickness of $60 \mathrm{~nm}$.

For the interpretation of these data we propose a toy model based on the following assumption: the parts of $\mathrm{CoO} / \mathrm{Co}$ bilayer deposited at the lateral surface of the $\mathrm{ZnO}$ grains experience the external magnetic field $\mathbf{H}_{\mathrm{ex}}$ at randomly distributed directions, as illustrated in the cartoon of figure 4 for the side view line-section of a single $\mathrm{ZnO}$ grain.

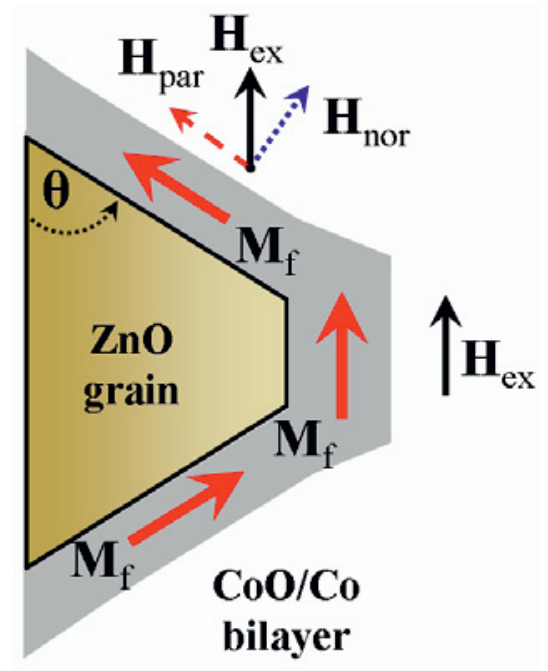

Fig. 4. The cartoon depicts, in side view, the misalignment observed between the local magnetization, $\mathbf{M}_{\mathbf{f}}$ of the $\mathrm{CoO} / \mathrm{Co}$ bilayer and the applied magnetic field, $\mathbf{H}_{\mathbf{e x}}$ at the neighbourhood of a $\mathrm{ZnO}$ grain due to the rough landscape. The thin solid arrow is the external magnetic field, $\mathbf{H}_{\text {ex }}$. The thick solid arrows depict the magnetization, $\mathbf{M}_{\mathbf{f}}$, that lies in-layer at the lateral sides of the $\mathrm{ZnO}$ grain. The dashed and dotted arrows represent the parallel and normal components of $\mathbf{H}_{\mathrm{ex}}$ to the local magnetization, $\mathbf{M}_{\mathbf{f}}$ of the $\mathrm{CoO} / \mathrm{Co}$ bilayer, respectively. The scheme refers to the parallel magnetic field-sample configuration.

Obviously, the rougher the $\mathrm{ZnO}$ landscape, the more randomly distributed is the direction between the local magnetization $\mathbf{M}_{\mathbf{f}}$ of the $\mathrm{CoO} / \mathrm{Co}$ structure and the external magnetic field $\mathbf{H}_{\mathrm{ex}}$. Referring to the $\mathrm{ZnO}$ substrates, representative AFM images of the surface landscape are shown in figures 5(a)-5(c) for $\mathrm{ZnO}$-film $(200 \mathrm{~nm})$, and ZnO-bulk sintered at $\mathrm{T}_{\sin }=800^{\circ} \mathrm{C}$ $\left(<\mathrm{S}_{\mathrm{a}}>=120 \mathrm{~nm}\right)$ and $\mathrm{T}_{\sin }=1400^{\circ} \mathrm{C} \quad\left(<\mathrm{S}_{\mathrm{a}}>=840 \mathrm{~nm}\right)$, respectively.

The overall decrease of both $\mathrm{H}_{\text {shift }}{ }^{\mathrm{EB}}$ and $\mathrm{H}_{\mathrm{c}}{ }^{\mathrm{EB}}$ upon increase of SR (IR) observed in both $10 \mathrm{~nm}$ and $60 \mathrm{~nm}$ thin Co films can be ascribed to the competition between the intrinsic mechanism of shape anisotropy that 'locks' $\mathbf{M}_{\mathbf{f}}$ in-layer [12,13] and the extrinsic cause of the applied magnetic field $\mathbf{H}_{\mathbf{e x}}$ that tries to bias $\mathbf{M}_{\mathbf{f}}$ by rotating it along its direction. We propose that the key parameter for the decrease of both $\mathrm{H}_{\text {shift }}{ }^{\mathrm{EB}}$ and $\mathrm{H}_{\mathrm{c}}{ }^{\mathrm{EB}}$ upon increase of SR (IR) observed in our data is the misalignment between $\mathbf{M}_{\mathbf{f}}$ and $\mathbf{H}_{\mathbf{e x}}$ due to the morphologically rough landscape of 
the $\mathrm{ZnO}$ substrate as clearly illustrated by the AFM data presented in figures 5(a)-5(c), in top view. The overall concept is schematically illustrated in figure 4 , in side view, for the parallel magnetic field-sample configuration; the parts of the $\mathrm{CoO} / \mathrm{Co}$ bilayer that is deposited at the lateral surfaces of the $\mathrm{ZnO}$ grains possess a magnetization vector, $\mathbf{M}_{\mathbf{f}}$ that since it is 'locked' inlayer due to the shape anisotropy $[12,13]$, it experiences the external magnetic field $\mathbf{H}_{\mathbf{e x}}$ at a misalignment angle $\theta$. As a consequence, the biasing potential of $\mathbf{H}_{\mathbf{e x}}$ is weakened due to its effectively smaller value $\mathbf{H}_{\mathbf{e x}}{ }^{\text {ef }}=\mathbf{H}_{\mathrm{par}}=\mathbf{H}_{\mathrm{ex}} \cos \theta$. Obviously, since the misalignment angle between $\mathbf{M}_{\mathbf{f}}$, that is 'directionally random' and $\mathbf{H}_{\mathbf{e x}}$, that is 'directionally oriented' increases with SR (IR), the biasing potential of $\mathbf{H}_{\mathbf{e x}}$ on $\mathbf{M}_{\mathbf{f}}$ should decrease. Thus, both relative macroscopic parameters $\mathrm{H}_{\text {shift }}{ }^{\mathrm{EB}}$ and $\mathrm{H}_{\mathrm{c}}{ }^{\mathrm{EB}}$ should also decrease, as observed in our data. The same explanation can be applied for the normal magnetic fieldsample configuration.
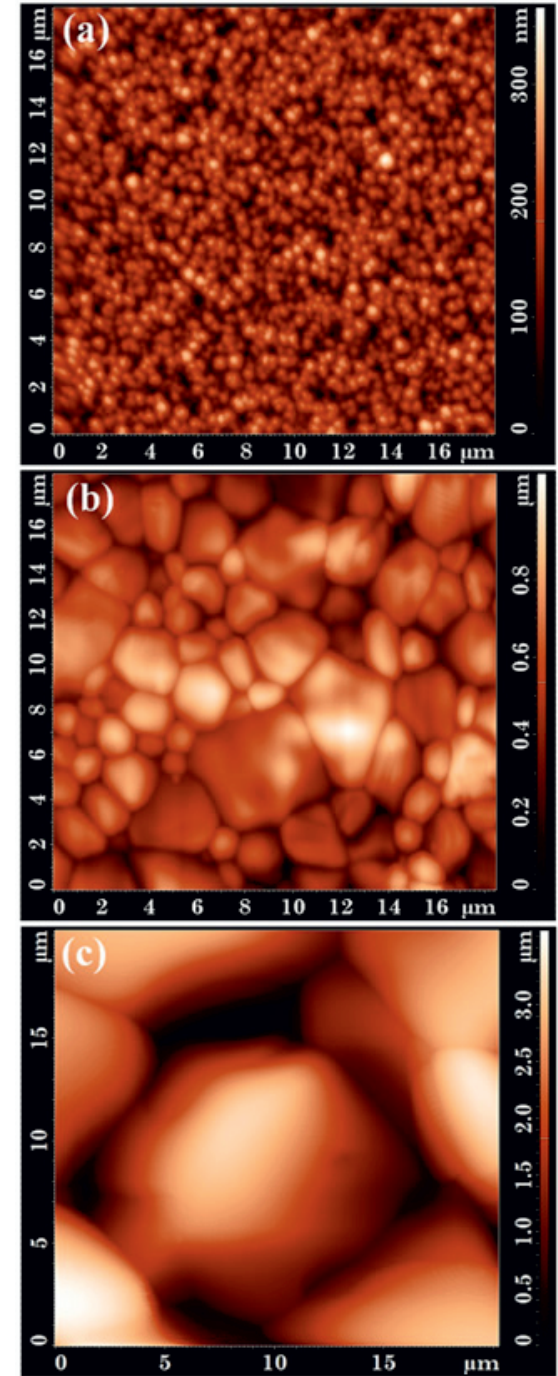

Fig. 5. Representative AFM images, in top view $\left(20 \times 20 \mu \mathrm{m}^{2}\right)$, of $\mathrm{ZnO}$ (a) film and bulk sintered at (b) $\mathrm{T}_{\sin }=800^{\circ} \mathrm{C}$ and (c) $\mathrm{T}_{\sin }=1400^{\circ} \mathrm{C}$.

Except for the issues discussed above additional mechanisms can be at play making the overall situation even more complicate. For instance, by using simple geometric arguments and algebraic calculations it is easy to show that the thickness of the deposited $\mathrm{CoO} / \mathrm{Co}$ bilayer is strongly modulated by the rough landscape of the $\mathrm{ZnO}$ substrates; the nominal thickness, $\mathrm{t}_{\text {nom }}$ is only preserved at the areas that during the deposition are normal to the incident plasma, while at the lateral surfaces of the $\mathrm{ZnO}$ grains the effective thickness, $t_{\text {eff }}$ is strongly reduced following a $t_{\text {eff }}=t_{\text {nom }} \cos \theta$ law, where $\theta$ is the angle shown in figure 4 that varies within $0^{\circ}$ and $90^{\circ}$. The effective reduction of the Co thickness farther promotes the shape anisotropy mechanism, ultimately weakening the bias potential of the applied magnetic field. The theoretical investigation of this additional mechanism is in progress.

\section{Conclusions}

In thin $\mathrm{CoO} / \mathrm{Co}$ bilayers deposited on rough $\mathrm{ZnO}$ substrates the magnetization, $\mathbf{M}_{\mathbf{f}}$ of Co is 'locked' mainly in-layer due to shape anisotropy; thus, it is forced to follow the morphologically rough landscape of the $\mathrm{ZnO}$ substrate. As a consequence, misalignment is observed between $\mathbf{M}_{\mathbf{f}}$, that is 'directionally random', and the biasing field $\mathbf{H}_{\mathbf{e x}}$, that is 'directionally oriented'. This weakens the biasing potential of $\mathbf{H}_{\mathbf{e x}}$ on $\mathbf{M}_{\mathbf{f}}$ and reduces the relative macroscopic parameters $\mathrm{H}_{\text {shift }}{ }_{\mathrm{EB}}$ amd $\mathrm{H}_{\mathrm{c}}^{\mathrm{EB}}$. The effect gets pronounced with the increase of SR (IR).

\section{Acknowledgments}

M. Zeibekis acknowledges the A.G. Leventis Foundation for support through a scholarship.

\section{References}

1. J. Nogues, I.K. Schuller, J. Magn. Magn. Mater. 192, 203 (1999)

2. M.N. Baibich, J.M. Broto, A. Fert, F. Nguyen Van Dau, F. Petroff, Phys. Rev. Lett. 61, 2472 (1988)

3. J. Daughton J. Brown, E. Chen, R. Beech, A. Pohm, W. Kude, IEEE Trans. Magn. 30, 4608 (1994)

4. M. Dax, Semicond. Int. 20, 84 (1997)

5. G.A. Prinz, Science 282, 1660 (1998)

6. N.M. Nemes et al., Phys. Rev. B 81, 024512 (2010)

7. D. Stamopoulos, E. Manios, M. Pissas, Supercond. Sci. Technol. 20, 1205 (2007)

8. D. Stamopoulos, E. Manios, M. Pissas, Phys. Rev. B 75, 014501 (2007)

9. D. Stamopoulos, E. Manios, M. Pissas, Exchange Biased and Plain Superconducting Ferromagnetic Layered Hybrids (Nova Science Publishers Inc, New York, USA, 2010)

10. B.D. Schrag et al., Appl. Phys. Lett. 77, 2373 (2000)

11. S. Demokritov et al., Phys. Rev. B 49, 720 (1994)

12. M. Hehn, S. Padovani, K. Ounadjela, J.P. Bucher, Phys. Rev. B 54, 3428 (1996)

13. J. Brandenburg, R. Huhne, L. Shultz, V. Neu, Phys. Rev. B 79, 054429 (2009) 